\title{
COPD and osteoporosis: something more than a comorbidity
}

\author{
Claudio Tantucci
}

Published online: 12 May 2012

(C) Springer Science+Business Media, LLC 2012

Chronic obstructive lung disease (COPD) is a progressive functional disorder of the respiratory system with high prevalence in the older population and is presently the fourth cause of mortality and supposed to be the third in the 2020 in the world [1]. Large part of COPD morbidity and mortality is due to extra-pulmonary effects rather than pulmonary consequences of this disorder, mainly when the airway obstruction is mild or moderate [2]. Among the extra-pulmonary effects, osteopenia and osteoporosis, as a consequence of a loss of bone mineral density (BMD), have been widely recognized as a major comorbidity in COPD patients [3].

While age, female sex and smoking are common pathogenetic factors, other factors such as reduced daily physical activity, malnutrition, low body mass index, hypogonadism, vitamin D deficiency, chronic renal insufficiency, chronic hypoxemia, and drugs (especially corticosteroids), due to COPD occurrence and progression, have been invoked to explain such a frequent association [4]. However, the presence of a low BMD even in COPD patients with milder airflow obstruction in whom the above mentioned, associated risk factors are less prevalent, may suggest another link between these two conditions.

In this article of the Journal, Liang and Feng add evidence to the belief that low-grade systemic inflammation, frequently observed in clinically stable COPD patients, could be the main underlying mechanism able to explain this relationship [5]. In this respect, the frequent

C. Tantucci $(\bowtie)$

Department of Medical and Surgical Sciences, University of Brescia, Brescia, Italy

e-mail: tantucci@med.unibs.it coexistence of COPD and metabolic syndrome [6] may increase the systemic burden of pro-inflammatory cytokines due to their overproduction by the increased visceral adipose tissue mass.

More than $60 \%$ of their COPD patients had osteopenia (34\%) and osteoporosis (29\%). Using the plasma level of CRP, IL-6 and TNF- $\alpha$ as surrogate marker of systemic inflammation, the authors found significantly higher levels of these cytokines in those COPD patients with abnormal BMD. Moreover, the risk of a low BMD was significantly increased by a factor 3 in the presence of elevated degree of systemic inflammation and only the levels of TNF- $\alpha$ and IL-6 were retained as independent predictors at a multivariate logistic analysis for a low BMD [5].

Two relevant facts clearly emerge from this study: again the high prevalence of osteopenia and osteoporosis, when properly assessed by dual-energy X-ray absorptiometry, in COPD patients showing moderate-to-severe airway obstruction and the strong relationship between the presence of COPD-related systemic inflammation and low BMD, even after adjustment for age, sex, use of inhaled corticosteroids (ICS), and severity of airflow obstruction.

Although the influence of other potential risk factors, particularly the daily physical activity that can be markedly reduced also in the mild-to-moderate COPD patients, was not taken into account, it is important to be aware that the severity of airway obstruction and the use of ICS are less strongly associated with the loss of BMD, than the increased plasma level of pro-inflammatory cytokines.

Based on a cross-sectional analysis, however, this study cannot determinate a causal relationship between lowgrade systemic inflammation and development of both osteopenia and osteoporosis in patients with COPD.

The nature of the low-grade systemic inflammation in COPD is still debated, but the significant BMD 
improvement 1 year after lung volume reduction surgery in severe emphysematous COPD patients as compared with a similar control group of COPD patients treated with respiratory rehabilitation, suggests that mechanical derangement of lung structure because of air trapping and parenchymal destruction might be pathogenetically relevant in sustaining an overall chronic inflammatory response and its consequences [7].

In any case, the high prevalence of osteopenia, and mostly, osteoporosis in COPD, as confirmed also in the study of Liang and Feng, indicates the necessity of carefully assessing BMD in COPD patients, at least in those with other associated risk factors, to avoid the most common clinical sequela of osteoporosis represented by the vertebral compression fracture [8]. This is even more relevant considering that for the same BMD loss, these kind of fractures are more frequently seen in COPD patients with severe-to-very severe disorder than in general population, possibly because during exacerbations prolonged cough, burst of systemic oral corticosteroids, accompanied by a sharp increase of systemic inflammation, may act as adjunctive negative stress factors especially in the thoracic vertebral bone. It is not useless to remember that in average for each thoracic vertebral fracture a $9 \%$ fall in forced vital capacity (FVC) can be expected [9]. Since forced expiratory volume in the first second $\left(\mathrm{FEV}_{1}\right)$ results from $\mathrm{FEV}_{1} / \mathrm{FVC} \times \mathrm{FVC}$, any FVC decrease implies a $\mathrm{FEV}_{1}$ decrease, aggravating the airflow reduction in COPD patients in a sort of vicious circle that could be easily prevented [10].

\section{References}

1. K.F. Rabe, S. Hurd, A. Anzueto, P.J. Barnes, S.A. Buist, P. Calverley, Y. Fukuchi, C. Jenkins, R. Rodriguez-Roisin, C. Weel, J. Zelinski, Global initiative for chronic obstructive lung disease, global strategy for the diagnosis, management and prevention of chronic obstructive pulmonary disease: GOLD executive summary. Am. J. Respir. Crit. Care Med. 176, 532-555 (2007)

2. P.M.A. Calverley, J.A. Anderson, B. Celli, G.T. Ferguson, C.R. Jenkins, P.W. Jones, J.C. Yates, J. Vestbo, Efficacy of salmeterol and fluticasone propionate on mortality in chronic obstructive lung disease. N. Engl. J. Med. 356, 775-789 (2007)

3. G.T. Ferguson, P.M.A. Calverely, J.A. Anderson, C.R. Jenkins, P.W. Jones, R.L. Willits, J.C. Yates, J. Vestbo, B. Celli, Prevalence and progression of osteoporosis in patients with COPD. Chest 136, 1456-1465 (2009)

4. A. Jebouck, S. Boonen, M. Decramer, W. Janssens, COPD, bone metabolism and osteoporosis. Chest 139, 648-657 (2011)

5. B. Lind, Y. Feng, The association of low bone mineral density with systemic inflammation in clinically stable COPD. Endocrine (2012). doi:10.1007s12020-011-9583

6. H. Watz, B. Waschki, A. Kristen, K.-C. Muller, G. Kretschmar, T. Meyer, O. Holz, H. Magnussen, The metabolic syndrome in patients with chronic bronchitis and COPD. Chest 136, 1039-1104 (2009)

7. T.C. Mineo, V. Ambrogi, D. Mineo, A. Fabbri, E. Fabbrini, R. Massoud, Bone mineral density improvement after lung volume reduction surgery for severe emphysema. Chest 127, 1960-1966 (2005)

8. S.R. Majumdar, C. Villa-Roel, K.J. Lyons, B.H. Rowe, Prevalence and predictors of vertebral fracture in patients with chronic obstructive pulmonary disease. Resp. Med. 104, 260-266 (2010)

9. R.A. Harrison, K. Siminoski, D.B. Vethanayagam, S.R. Majumdar, Osteoporosis related kyphosis and impairments in pulmonary function: a systematic review. J. Bone Miner. Res. 22, 247-257 (2007)

10. G. Mazziotti, J. Bilezikian, E. Canalis, D. Cocchi, A. Giustina, New understanding and treatment for osteoporosis. Endocrine 41, 58-69 (2012) 